\title{
Electrical resistivity of nanoporous gold modified with thiol self- assembled monolayers
}

\section{AUTHOR(S):}

Hakamada, Masataka; Kato, Naoki; Mabuchi, Mamoru

\section{CITATION:}

Hakamada, Masataka ...[et al]. Electrical resistivity of nanoporous gold modified with thiol self-assembled monolayers. Applied Surface Science 2016, 387: 1088-1092

\section{ISSUE DATE:}

2016-11-30

URL:

http://hdl.handle.net/2433/237624

\section{RIGHT:}

(c) 2016. This manuscript version is made available under the CC-BY-NC-ND 4.0 license

http://creativecommons.org/licenses/by-nc-nd/4.0/; The full-text file will be made open to the public on 30 November 2018 in accordance with publisher's 'Terms and Conditions for Self-Archiving'.; この論文は出版社版でありません。引 用の際には出版社版をご確認ご利用ください。; This is not the published version. Please cite only the published version. 


\section{Electrical resistivity of nanoporous gold modified with thiol self-assembled monolayers}

Masataka Hakamada*, Naoki Kato ${ }^{1}$, Mamoru Mabuchi

Department of Energy Science and Technology, Graduate School of Energy Science, Kyoto University, Yoshidahonmachi, Kyoto, 606-8501 Japan

*Corresponding author. E-mail: hakamada.masataka.3x@kyoto-u.ac.jp (M. Hakamada), katou.naoki.75w@st.kyoto-u.ac.jp (N. Kato), mabuchi@energy.kyoto-u.ac.jp (M. Mabuchi).

1 Present address: Tanaka Kikinzoku Kogyo K. K., Japan. 


\section{Abstract}

The electrical resistivity of nanoporous gold (NPG) modified with thiol self-assembled monolayers (SAMs) has been measured at $298 \mathrm{~K}$ using a four-probe method. We found that the adsorption of thiol SAMs increases the electrical resistivity of NPG by up to 22.2\%. Dependence of the electrical resistivity on the atmosphere (air or water) was also observed in SAMs-modified NPG, suggesting that the electronic states of the tail groups affect the electrons of the binding sulfur and adjacent surface gold atoms. The present results suggest that adsorption of thiol molecules can influence the behavior of the conducting electrons in NPG and that modification of NPG with SAMs may be useful for environmental sensing.

Keywords:

Nanoporous gold; self-assembled monolayers; thiols; electrical resistivity 


\section{Introduction}

Adsorption of various molecules on the surface of a substrate can be detected by monitoring the electrical properties of the substrate material [1-7]. The substrate materials of these "chemiresistors" [2] should possess both nanostructures with high surface area and electrical conductivity. For example, adsorption of oxygen on carbon nanotubes $[3,4]$ and protein on silicon nanowires $[5,6]$ can be successfully detected. In particular, Kisner et al. [7] reported that the adsorption of self-assembled monolayers (SAMs) can be detected better on gold nanowires than on bulk gold, suggesting that the electrical resistance is strongly influenced by free electron scattering at the surface when the size of the metallic materials is smaller than the typical length of the mean free path of electrons $[8,9]$.

Nanoporous gold (NPG) has open porous structures with pores and ligaments in the nanometer size range and can be fabricated by dealloying or selective dissolution of less noble element from binary alloys in an electrolyte [10,11]. Monolithic nanoporous metals with high specific surface areas exhibit adsorption-driven macroscopic actuation in aqueous and gaseous environments [12-15]. The sensitivity of NPG to the atmosphere suggests that the electrical properties of NPG are also altered by adsorption. In addition, the conditions used for dealloying and post-treatment determine the pore and ligament 
sizes of NPG; under conditions that suppresses the self-organization of gold atoms, the pore and ligament sizes are as small as the mean free path of electrons. Consequently, the electrical properties of NPG are expected to be sensitive to the adsorption of chemical species.

Considering these factors, we compare the change in electrical properties of NPG resulting from the adsorption of thiol SAMs with different tail groups. The effects of the atmosphere (air and water) and the thiol concentration in the ethanolic solution are also examined.

\section{Materials and Methods}

NPG films were fabricated by dealloying of Au0.25Ago.75/pure-Au stacked film sputtered on an insulating glass substrate. The schematic illustration of the fabrication procedure is shown in Fig. 1a. NPG films were produced by dealloying of the prepared film sample in 70 mass $\% \mathrm{HNO}_{3}$ for $24 \mathrm{~h}$ at $253 \mathrm{~K}$ under free corrosion conditions (without anodic electrochemical potential). The low dealloying temperature of $253 \mathrm{~K}$ was adopted for finer pores and ligaments of the resulting NPG [16]. First, a 70-nm-thick gold adhesion layer was sputtered on the glass and then a 140-nm-thick Au0.25Ag0.75 layer was sputtered on the gold adhesion layer by radio-frequency magnetron sputtering. The 
underlying gold adhesion layer is necessary to prevent fragmentation of NPG layer. A metallic mask (Fig. 1b) was used for the fabrication of a circuit of sputtered film that is suitable for electrical resistivity measurements using a four-probe method. The dealloyed samples were thoroughly washed with distilled water to remove residual acid. For comparison, a 140-nm-thick flat gold (FG) film without a nanoporous structure was also prepared by sputtering on a glass substrate. The thickness of the sputtered films was confirmed using a probe-type step profiler (Dektak150 by ULVAC Corp.).

(a)



2. Dealloying

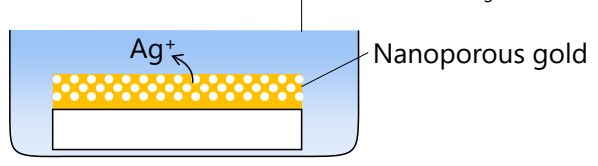

(b)

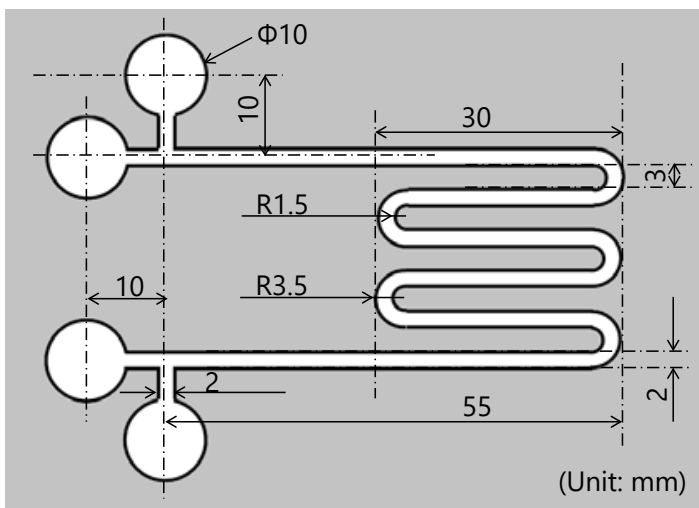

Fig. 1. (a) Schematic illustration of the fabrication of nanoporous gold thin film for measurement of electrical resistivity. (b) Metallic mask for sputtering.

Propanethiol ( $\mathrm{HS}\left(\mathrm{CH}_{2}\right)_{2} \mathrm{CH}_{3}$, PT, purity >95\%), mercaptopropionic acid $\left(\mathrm{HS}\left(\mathrm{CH}_{2}\right)_{2} \mathrm{COOH}, \mathrm{MPA}\right.$, purity >97\%) and cysteamine $\left(\mathrm{HS}\left(\mathrm{CH}_{2}\right)_{2} \mathrm{NH}_{2}, \mathrm{CA}\right.$, purity >97\%) were purchased from a commercial supplier (Wako Pure Chemical Industries Ltd., Japan). 0.1 and $10 \mathrm{mmol} / \mathrm{L}$ solutions of the thiols were prepared in absolute ethanol 
(purchased from Nacalai Tesque, Inc., Japan). The NPG and FG samples were modified with SAMs by immersing the fabricated gold samples in the ethanolic solutions of the thiols for $24 \mathrm{~h}$. Afterwards, the samples were removed from the solutions and washed with absolute ethanol and distilled water.

The electrical resistivity of the NPG and FG samples was measured at $298 \pm 0.5$ $\mathrm{K}$ by a direct current four-probe method in air and in ion-exchanged and distilled water. The electrical resistivity of the NPG layers was calculated assuming that the two layers have a parallel connection for electron conductivity [17]. The change in the electrical resistivity $\left(\Delta \rho / \rho_{0}\right)$ of NPG resulting from SAMs modification was then calculated using the following equation:

$$
\frac{\Delta \rho}{\rho_{0}}=\frac{\rho_{\mathrm{SAM}}-\rho_{0}}{\rho_{0}}
$$

where $\rho_{0}$ is the electrical resistivity of NPG or FG without modification by SAMs measured in air and $\rho$ SAM is the electrical resistivity of SAMs-modified NPG or FG measured in air or in ion-exchanged and distilled water. The thermal equilibrium was confirmed by monitoring the electric potential during the measurements. To check reproducibility, three or four identical samples were fabricated and their electrical resistivity was measured.

The microstructure of the samples was observed by scanning electron 
microscopy (SEM). To estimate the surface element composition of the samples modified with SAMs, X-ray photoelectron spectroscopy (XPS) was performed with a standard Mg $\mathrm{K} \alpha$ source $(200 \mathrm{~W})$ at a takeoff angle of $45^{\circ}$. The samples were fabricated by the sputtering process described above (without a metal mask), dealloying and modification with SAMs $(10 \mathrm{mmol} / \mathrm{L})$ on $11 \times 11 \mathrm{~mm}^{2}$ cover glass.

\section{Results and discussion}

The microstructure of the fabricated NPG is shown in Fig. 2. A nanoporous structure with an average ligament diameter of $24 \mathrm{~nm}$ (calculated from the SEM images of 100 ligaments) was observed. The electrical resistivity of the NPG at room temperature was measured to be $426 \mathrm{n} \Omega \mathrm{m}$, which is in agreement with other experimental data [1821]. 


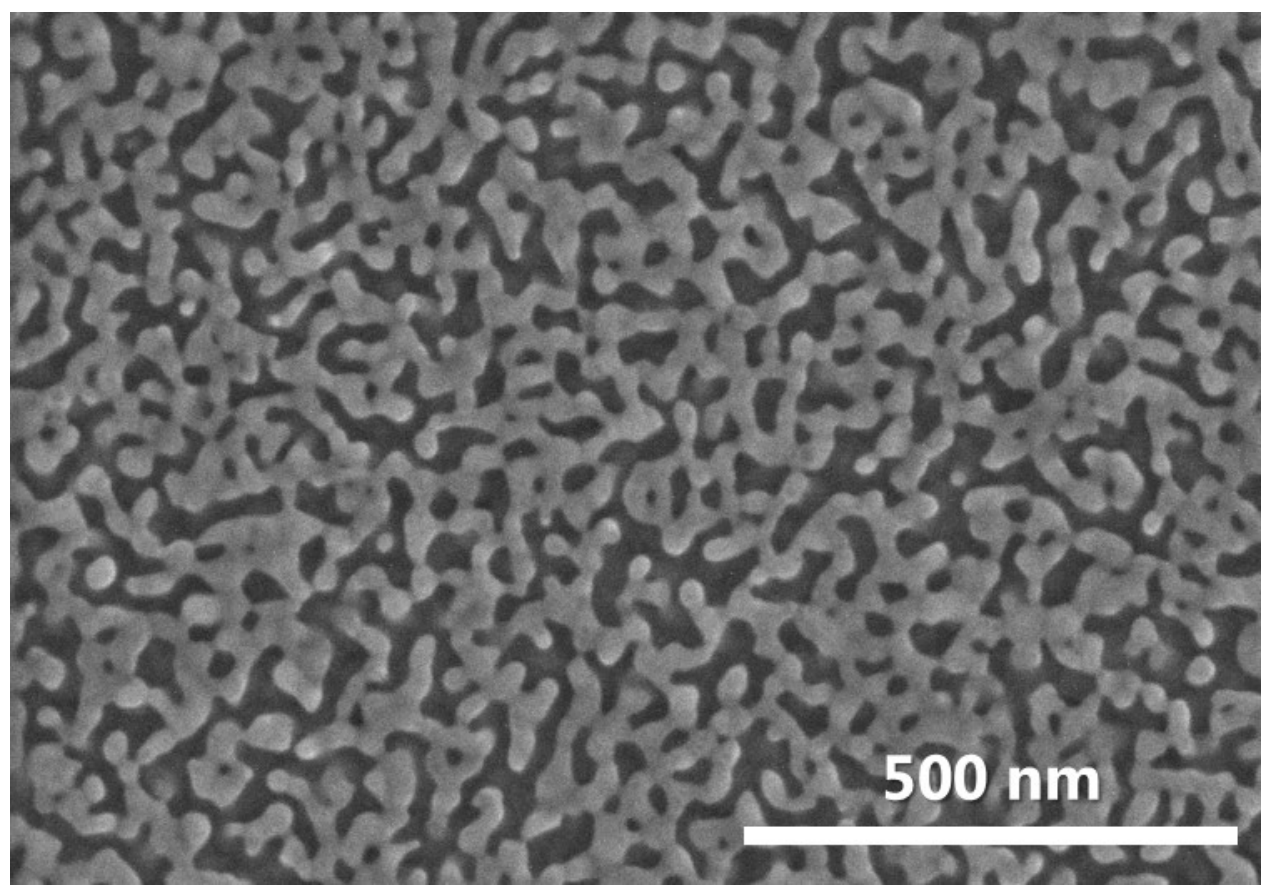

Fig. 2. Scanning electron microscopy image of nanoporous gold.

The changes in the electrical resistivity $\left(\Delta \rho / \rho_{0}\right)$ of NPG and FG as a result of modification with SAMs are shown in Figs. 3-5. Overall, the $\Delta \rho / \rho_{0}$ of NPG was clearly positive and much higher than that of FG, irrespective of the types of SAMs used. Under both air and water atmospheres, a higher SAMs concentration in the ethanolic solution resulted in a larger $\Delta \rho / \rho_{0}$ of NPG, whereas the concentration dependence of the $\Delta \rho / \rho_{0}$ of FG was not clear. These results suggest that the electrical resistivity of NPG is very sensitive to the surface adsorption of the thiol SAMs. Moreover, $\Delta \rho / \rho_{0}$ of NPG in water was higher than in air for all three SAMs, whereby the largest $\Delta \rho / \rho_{0}(22.2 \%)$ of NPG modified by CA was followed by the $\Delta \rho / \rho_{0}$ of MPA and then PT. The atmosphere dependence (air or water) of $\Delta \rho / \rho_{0}$ of SAMs-modified NPG implies that the electrical 
properties of NPG depend not only on the surface adsorption of SAMs but also on the charged state of the functional tail groups in the molecular structures of the SAMs.

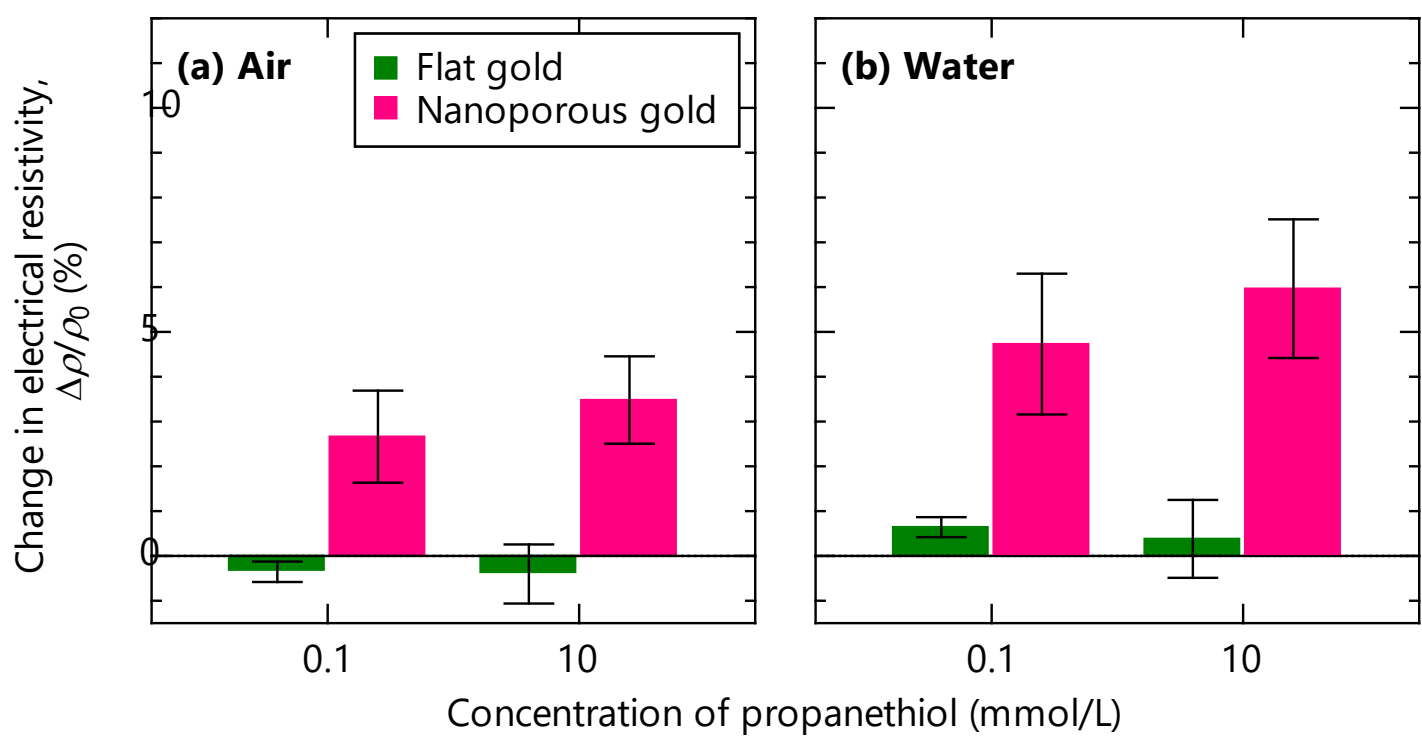

Fig. 3. Change in the electrical resistivity of flat gold and nanoporous gold modified by self-assembled monolayers of propanethiol.

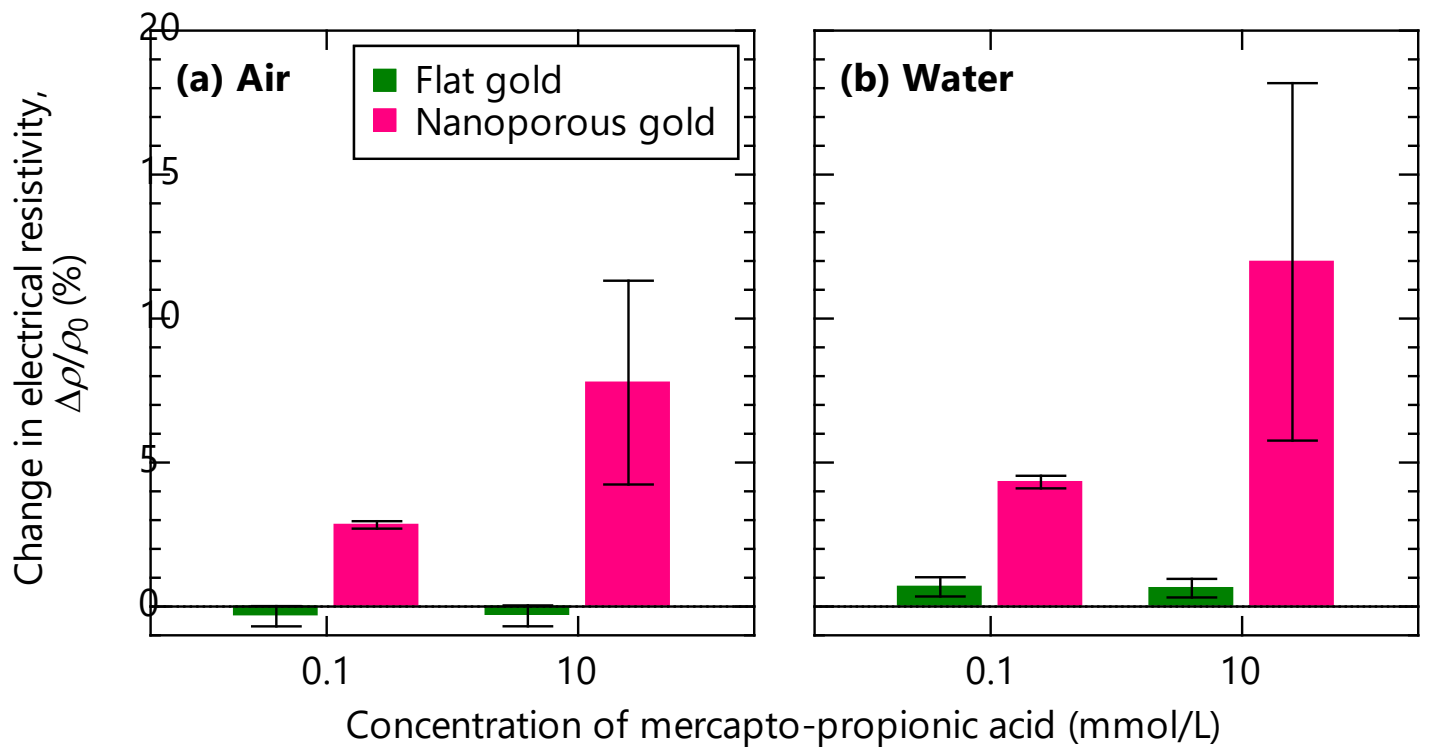

Fig. 4. Change in the electrical resistivity of flat gold and nanoporous gold modified by self-assembled monolayers of mercaptopropionic acid. 


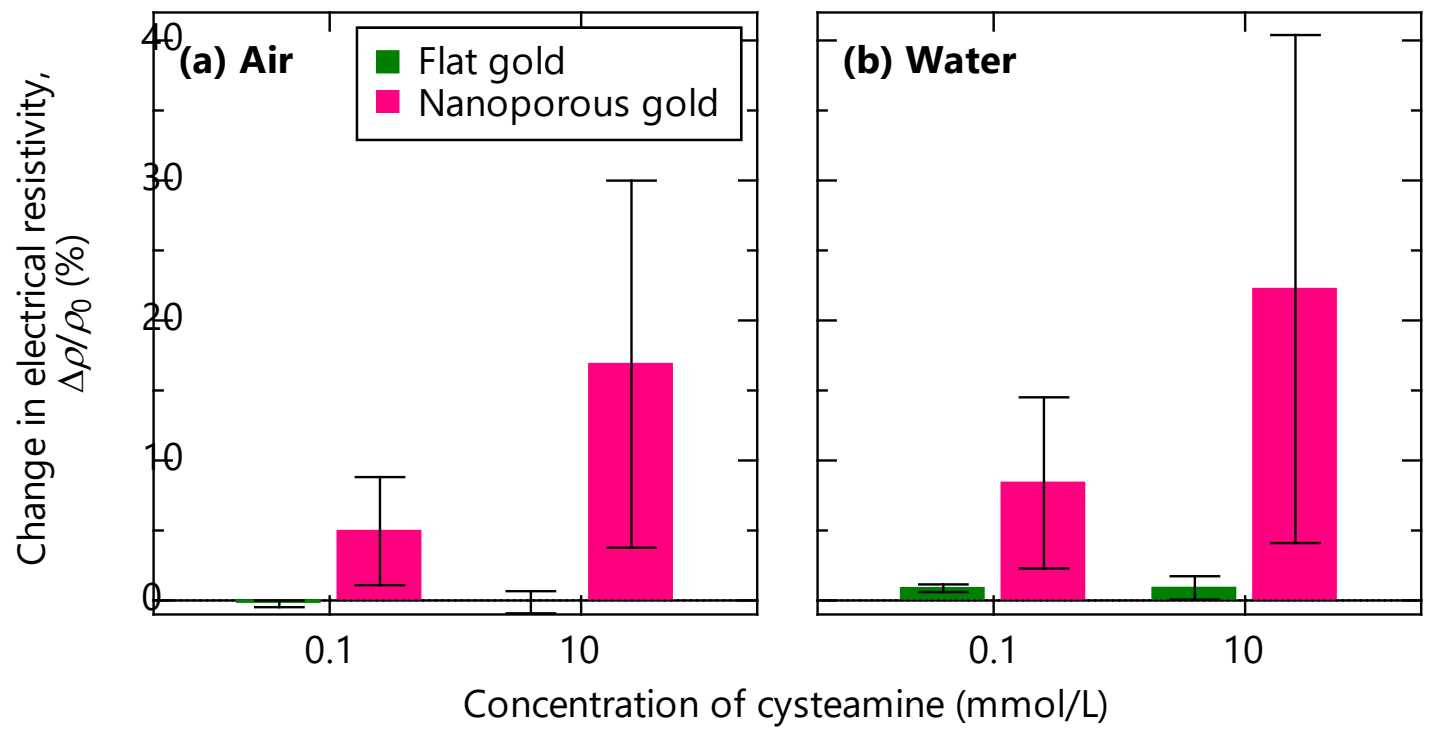

Fig. 5. Change in the electrical resistivity of flat gold and nanoporous gold modified by self-assembled monolayers of cysteamine.

To confirm the adsorption of SAMs on the sample surface and to estimate the surface coverage of the NPG with thiol molecules, XPS was performed on NPG samples modified with $10 \mathrm{mmol} / \mathrm{L}$ ethanolic solution of SAMs. The relative atomic compositions of sulfur to surface-existent gold, silver and sulfur atoms are plotted along the horizontal axis against $\Delta \rho / \rho_{0}$ in Fig. 6 (See Supplementary data for XPS profiles and calculation of atomic composition). The atomic composition of sulfur is a little higher than the surface coverage of the three thiols on Au (111) investigated mainly by scanning tunneling microscopy [22-24], although the difference is not significantly large. The highest coverage density by SAMs was obtained in the case of CA, followed by MPA and then PT. This tendency agrees with the order of $\Delta \rho / \rho_{0}$ of NPG modified with $10 \mathrm{mmol} / \mathrm{L}$ 
ethanolic solution of SAMs, as clearly shown in Fig. 6. That is, the electrical resistivity of NPG is roughly proportional to the amount of adsorbed thiol molecules.

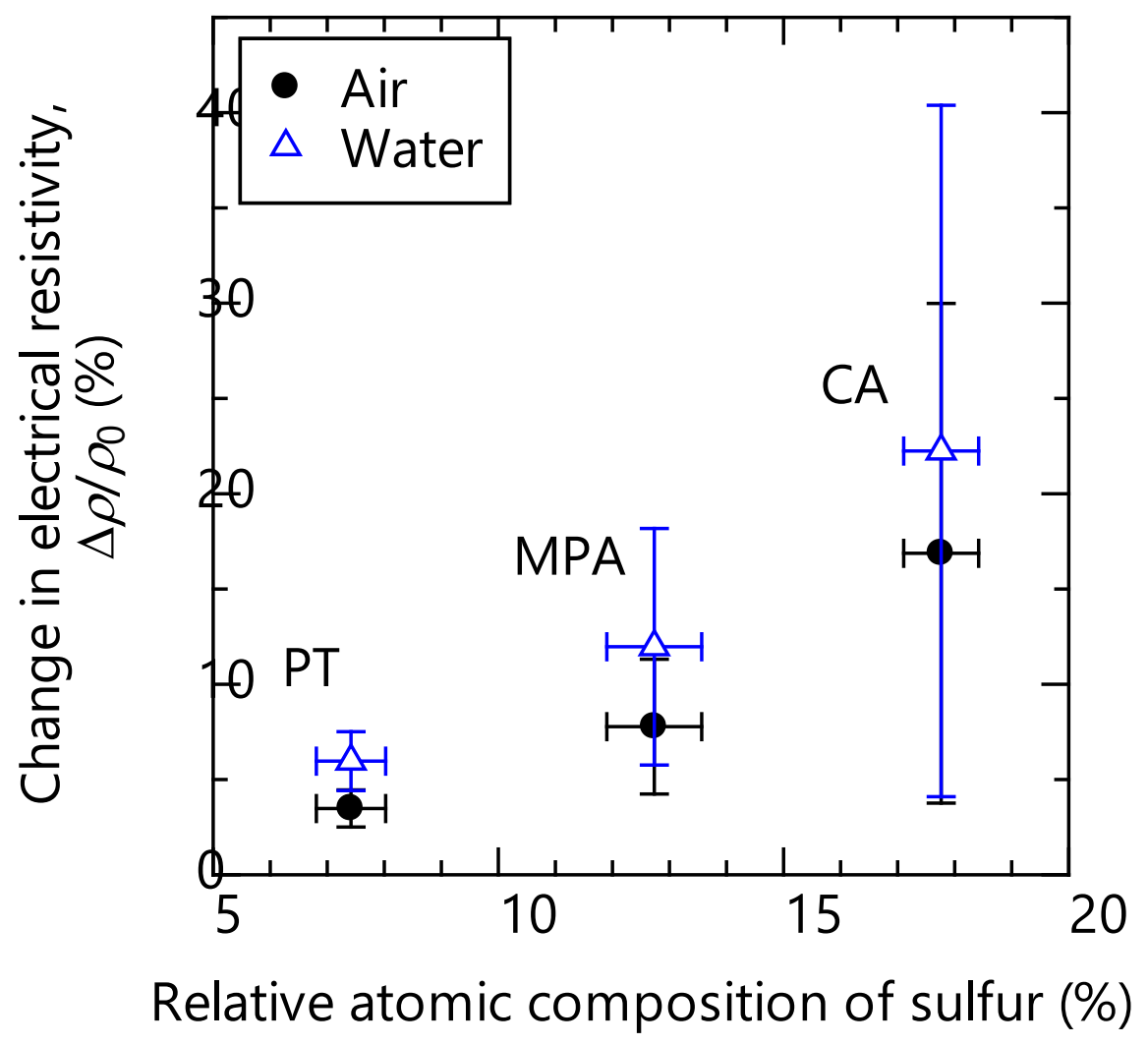

Fig. 6. Relationship between the relative atomic composition of sulfur and change in the electrical resistivity of nanoporous gold modified with $10 \mathrm{mmol} / \mathrm{L}$ propanethiol (PT), mercaptopropionic acid (MPA) and cysteamine (CA) in an ethanolic solution.

Kisner et al. [7] reported that the electrical resistance of an ultrathin gold nanowire (Au-NW, 2 nm in diameter) increases after modification with SAMs under both air and water atmospheres. The present results of the electrical resistivity of SAMsmodified NPG are in qualitative agreement with those in the study by Kisner et al. However, the magnitude of the increase in the electrical resistivity of NPG by SAMs 
modification was lower than that in Au-NW because the specific surface area (surfaceto-volume ratio) of NPG is lower than that of the Au-NW. The present NPG has ligaments with diameter of $24 \mathrm{~nm}$, which is 12 times larger than the diameter of the Au-NW (2 nm), resulting in a smaller specific surface area in NPG. By assuming that the ligaments in the NPG and nanowires in the study by Kisner et al. are columnar, the specific surface area of NPG is $1 / 12$ that of Au-NW. Table 1 compares the increase in the relative electrical resistivity of the present NPG $(\Delta \rho / \rho 0)$ with the relative electrical resistance $(\Delta R / R)_{\mathrm{Au}-\mathrm{NW}}$ of Au-NW. The ratio of $(\Delta R / R)_{\text {Au-NW }}$ to $\Delta \rho / \rho_{0}$ is roughly close to the specific surface area ratio (12) of Au-NW to NPG. This comparison also supports the finding that the $\Delta \rho / \rho_{0}$ detected in the present NPG is attributed to the surface adsorption of thiol SAMs.

The behavior of the conductive electron in a metal depends a lot on the electrons at the Fermi surface and the electrical conductivity is proportional to the density of states at the Fermi energy level [25]. It has also been reported that the thiol binding effect contributes to an increase in the electrical resistance of gold with reduced dimension by the change in the density of states at the Fermi energy level [26]. Hence, the adsorption of SAMs on the NPG increases the electrical resistivity.

The change in electrical properties owing to charge trapping by water adsorption at the surface has been observed $[27,28]$ and the effect of the acid-base chemistry of the 
functional groups of the SAMs on the interfacial electrical characteristics of gold electrodes has been reported $[29,30]$. These findings suggest that thiol binding causes a significant positive change in electrical resistivity of NPG.

The $\Delta \rho / \rho_{0}$ in NPG were always higher in water than in air. The influence of the atmosphere (air or water) was almost always larger in the MPA- and CA-modified NPG than in the PT-modified NPG. The tail of PT is nonpolar, whereas that of MPA and CA is polar because of the carboxyl $(-\mathrm{COOH})$ and amino $\left(-\mathrm{NH}_{2}\right)$ groups, respectively. Therefore, the polar tails of MPA and CA may interact with atmospheric water molecules, which has intrinsic polarity; and the electronic states of the head sulfur atoms and adjacent gold atoms may be influenced, resulting in the greater influence from the atmosphere, although the precise determination of protonation/deprotonation state, which may be conducted by analysis of current-voltage hysteresis [7], is not discussed here because of large data scattering (Figs. 3-6). In other words, the results shown here are at early stage of investigation; detailed atomistic phenomena such as protonation/deprotonation state and monolayer/multilayer aspects at SAM on NPG will be clarified by further experiments and/or simulation. Thinner underlying bulk gold layer, or even absence of underlying layer is also desirable for better evaluation of the change in the electrical resistivity of NPG and application of NPG for sensing use, although the attempt has not 
succeeded at the present stage. The maximum/minimum concentration of thiol molecules that affects the change in electrical resistivity of NPG should be examined for practical application.

\section{Conclusions}

We have shown that the electrical resistivity of NPG film depends on the absorption of SAMs, whereas that of FG with similar thickness does not. The electrical resistivity of NPG was sensitive to the concentration and tail group of the thiol molecules. XPS elemental analyses of NPG and comparison of the present results with the electrical resistance change of Au-NW [7] imply that the adsorption of the thiol molecule on the ligaments in NPG increases the electrical resistivity. Furthermore, the change in electrical resistivity of NPG was greater in water than in air, which suggests that the atmosphere affects the electronic state of the head sulfur atom and adjacent gold atoms through the interaction between water and the tail of the thiol molecules. These results suggest that the adsorption of thiol molecules can influence the electric characteristics of NPG and that SAMs modification on NPG may be useful for environmental sensing. 


\section{Acknowledgements}

The author M. M. thanks the Japan Prize Foundation for the financial support.

Observations by SEM were conducted in the Research and Education Center of Advanced

Energy Science, Graduate School of Energy Science, Kyoto University.

Appendix A. Supplementary data

Supplementary data associated with this article can be found, in the online version, at $\{\mathrm{URL}\}$. 


\section{References}

[1] B.K. Duan, J. Zhang, P.W. Bohn, Conductance-based chemical sensing in metallic nanowires and metal-semiconductor nanostructures, Anal. Chem. 84 (2012) 2-8.

[2] I.S. Muratova, K.N. Mikhelson, Y.E. Ermolenko, A. Offenhäusser, Y. Mourzina, Chemiresistors based on ultrathin gold nanowires for sensing halides, pyridine and dopamine, Sens. Actuator B-Chem. 232 (2016) 420-427.

[3] P.G. Collins, K. Bradley, M. Ishigami, A. Zettl, Extreme oxygen sensitivity of electronic properties of carbon nanotubes, Science 287 (2000) 1801-1804.

[4] M. Shim, A. Javey, N.W.S. Kam, H. Dai, Polymer functionalization for air-stable ntype carbon nanotube field-effect transistors, J. Am. Chem. Soc. 123 (2001) 1151211513.

[5] G. Zheng, F. Patolsky, Y. Cui, W.U. Wang, C.M. Lieber, Multiplexed electrical detection of cancer markers with nanowire sensor arrays, Nature Biotechnol. 23 (2005) 1294-1301.

[6] Y. Cui, Q. Wei, H. Park, C.M. Lieber, Nanowire nanosensors for highly sensitive and selective detection of biological and chemical species, Science 293 (2001) 12891292. 
[7] A. Kisner, M. Heggen, D. Mayer, U. Simon, A. Offenhäusser, Y. Mourzina, Probing the effect of surface chemistry on the electrical properties of ultrathin gold nanowire sensors, Nanoscale 6 (2014) 5146-5155.

[8] E. H. Sondheimer, The mean free path of electrons in metals, Adv. Phys. 1 (1952) $1-42$.

[9] K. Fuchs, The conductivity of thin metallic films according to the electron theory of metals, Proc. Cambridge Philos. Soc. 34 (1938) 100-108.

[10]A. J. Forty, Corrosion micromorphology of noble metal alloys and depletion gilding, Nature 282 (1979) 597-598.

[11]J. Erlebacher, M.J. Aziz, A. Karma, N. Dimitrov, K. Sieradzki, Evolution of nanoporosity in dealloying, Nature 410 (2001) 450-453.

[12]D. Kramer, R.N. Viswanath, J. Weissmüller, Surface-stress induced macroscopic bending of nanoporous gold cantilevers, Nano Lett. 4 (2004) 793-796.

[13] J. Biener, A. Wittstock, L. A. Zepeda-Ruiz, M.M. Biener, V. Zielasek, D. Kramer, R. N. Viswanath, J. Weissmüller, M. Bäumer, A. V. Hamza, Surface-chemistry-driven actuation in nanoporous gold, Nat. Mater. 8 (2009) 47-51.

[14]H.-J. Jin, X.-L. Wang, S. Parida, K. Wang, M. Seo, J. Weissmüller, Nanoporous Au- 
Pt alloys as large strain electrochemical actuators, Nano Lett. 10 (2010) 187-194.

[15] M. Hakamada, S. Matsumura, M. Mabuchi, Electrochemical actuation of nanoporous $\mathrm{Ni}$ in $\mathrm{NaOH}$ solution, Mater. Lett. 70 (2012) 132-134.

[16]L.H. Qian, M.W. Chen, Ultrafine nanoporous gold by low-temperature dealloying and kinetics of nanopore formation, Appl. Phys. Lett. 91 (2007) 083105.

[17]K. Nakamura, S. Kikuchi, Resistivity of iron/copper multilayer materials, J. Jpn. Inst. Met. Mater. 72 (2008) 800-803 (in Japanese).

[18]R.W. Ertenberg, B. Andraka, Y. Takano, Prospects of porous gold as a lowtemperature heat exchanger for liquid and solid helium, Physica B 284-288 (2000) 2022-2023.

[19] T. Fujita, H. Okada, K. Koyama, K. Watanabe, S. Maekawa, M.W. Chen, Unusually small electrical resistance of three-dimensional nanoporous gold in external magnetic fields, Phys. Rev. Lett. 101 (2008) 166601.

[20]E. Seker, M. Reed, M. Utz, M.R. Begley, Flexible and conductive bilayer membranes of nanoporous gold and silicone: synthesis and characterization, Appl. Phys. Lett. 92 (2008) 154101. 
[21]R. Xia, J.L. Wang, R. Wang, X. Li, X. Zhang, X.Q. Feng, Y. Ding, Correlation of the thermal and electrical conductivities of nanoporous gold, Nanotechnology 21 (2010) 085703.

[22]J. Zhang, Q. Chi, J. Ulstrup, Assembly dynamics and detailed structure of 1propanethiol monolayers on $\mathrm{Au}(111)$ surfaces observed real time by in situ STM, Langmuir 22 (2006) 6203-6213.

[23] M. Petri, D.M. Kolb, U. Memmert, H. Meyer, Adsorption of mercaptopropionic acid onto Au(111) Part I. adlayer formation, structure and electrochemistry, Electrochim. Acta 49 (2003) 175-182.

[24] J. Zhang, A. Bilic ${ }^{-}$, J.R. Reimers, N.S. Hush, J. Ulstrup, Coexistence of multiple conformations in cycteamine monolayers on Au(111), J. Phys. Chem. B 109 (2005) 15355-15367.

[25]J.M. Ziman, Principles of the Theory of Solids, Cambridge University Press, Cambridge, 1964.

[26]A. Roy, T. Pandey, N. Ravishankar, A.K. Singh, Semiconductor-like sensitivity in metallic ultrathin gold nanowire-based sensors, J. Phys. Chem. C 118 (2014) 1867618682.

[27]W. Kim, A. Javey, O. Vermesh, Q. Wang, Y. Li, H. Dai, Hysteresis caused by water 
molecules in carbon nanotube field-effect transistors, Nano Lett. 3 (2003) 193-198.

[28]D. Wang, Y.L. Chang, Q. Wang, J. Cao, D.B. Farmer, R.G. Gordon, H. Dai, Surface chemistry and electrical properties of germanium nanowires, J. Am. Chem. Soc.

126 (2004) 11602-11611.

[29] C.P. Smith, H.S. White, Voltammetry of molecular films containing acid/base groups, Langmuir 9 (1993) 1-3.

[30]I. Burgess, B. Seivewright, R.B. Lennox, Electric field driven protonation/deprotonation of self-assembled monolayers of acid-terminated thiols, Langmuir 22 (2006) 4420-4428. 
Tables

Table 1. Change in the electrical resistivity of nanoporous gold (NPG) $\left(\Delta \rho / \rho_{0}\right)$ and electrical resistance of ultrathin gold nanowire (Au-NW) [7] $\left.\left((\Delta R / R)_{\text {Au-NW }}\right)\right)$ modified by SAMs with an ethanolic solution of 0.1 and $10 \mathrm{mmol} / \mathrm{L}$ organothiol species.

\begin{tabular}{lrrrrrr}
\hline \multirow{2}{*}{$10 \mathrm{mmol} / \mathrm{L}$} & \multicolumn{2}{c}{ Propanethiol } & \multicolumn{2}{c}{ Mercaptopropionic acid } & \multicolumn{2}{c}{ Cysteamine } \\
\cline { 2 - 7 } & \multicolumn{1}{c}{ Air } & \multicolumn{1}{c}{ Water } & \multicolumn{1}{c}{ Air } & Water & \multicolumn{1}{c}{ Air } & \multicolumn{1}{c}{ Water } \\
\hline$(\Delta R / R)_{\text {Au-NW [7] }(\%)}$ & 209.7 & 277.7 & 163.0 & 327.1 & 177.5 & 315.9 \\
$\Delta \rho / \rho_{0}$ (Present) (\%) & 3.5 & 6.0 & 7.8 & 12.0 & 16.9 & 22.2 \\
\hline Ratio & \multirow{2}{*}{60} & 47 & 21 & 27 & 11 & 14 \\
$($ Au-NW/NPG) & & & & & & \\
\hline
\end{tabular}

\begin{tabular}{lrrrrrr}
\hline \multirow{2}{*}{$0.1 \mathrm{mmol} / \mathrm{L}$} & \multicolumn{2}{c}{ Propanethiol } & \multicolumn{2}{c}{ Mercaptopropionic acid } & \multicolumn{2}{c}{ Cysteamine } \\
\cline { 2 - 8 } & \multicolumn{1}{c}{ Air } & \multicolumn{1}{c}{ Water } & \multicolumn{1}{c}{ Air } & Water & \multicolumn{1}{c}{ Air } & Water \\
\hline$(\Delta R / R)_{\text {Au-NW [7] }(\%)}$ & 44.4 & 115.3 & 42.0 & 22.9 & 22.4 & 68.2 \\
$\Delta \rho / \rho_{0}$ (Present) (\%) & 2.7 & 4.7 & 2.8 & 4.3 & 5.0 & 8.4 \\
\hline Ratio & 17 & 24 & 15 & 5 & 5 & 8 \\
$($ Au-NW/NPG) & & & & & & \\
\hline
\end{tabular}




\section{Figure captions}

Fig. 1. (a) Schematic illustration of the fabrication of nanoporous gold thin film for measurement of electrical resistivity. (b) Metallic mask for sputtering.

Fig. 2. Scanning electron microscopy image of nanoporous gold.

Fig. 3. Change in the electrical resistivity of flat gold and nanoporous gold modified by self-assembled monolayers of propanethiol.

Fig. 4. Change in the electrical resistivity of flat gold and nanoporous gold modified by self-assembled monolayers of mercaptopropionic acid.

Fig. 5. Change in the electrical resistivity of flat gold and nanoporous gold modified by self-assembled monolayers of cysteamine.

Fig. 6. Relationship between the relative atomic composition of sulfur and change in the electrical resistivity of nanoporous gold modified with $10 \mathrm{mmol} / \mathrm{L}$ propanethiol (PT), mercaptopropionic acid (MPA) and cysteamine (CA) in an ethanolic solution. 


\section{Supplementary data for:}

\section{Electrical resistivity of nanoporous gold modified with thiol self- assembled monolayers}

Masataka Hakamada*, Naoki Kato ${ }^{1}$, Mamoru Mabuchi

Department of Energy Science and Technology, Graduate School of Energy Science, Kyoto University, Yoshidahonmachi, Kyoto, 606-8501 Japan

* Corresponding author. E-mail address: hakamada.masataka.3x@kyoto-u.ac.jp (M. Hakamada).

1 Present address: Tanaka Kikinzoku Kogyo K. K., Japan. 


\section{X-ray photoelectron spectroscopy (XPS) of thiol-modified nanoporous gold}

Figure S1 shows XPS profiles of nanoporous gold modified by $10 \mathrm{mmol} / \mathrm{L}$ ethanolic solution of propanethiol (PT), mercaptopropionic acid (MPA) and cysteamine (CA). The elemental surface compositions were calculated by the peak area from $\mathrm{Au} 4 \mathrm{f}, \mathrm{Ag} 3 \mathrm{~d}$ and S 2p, where background was corrected by Shirley method. (The contribution of peak C 1s is eliminated because of possible contamination of carbonaceous species during operation.)

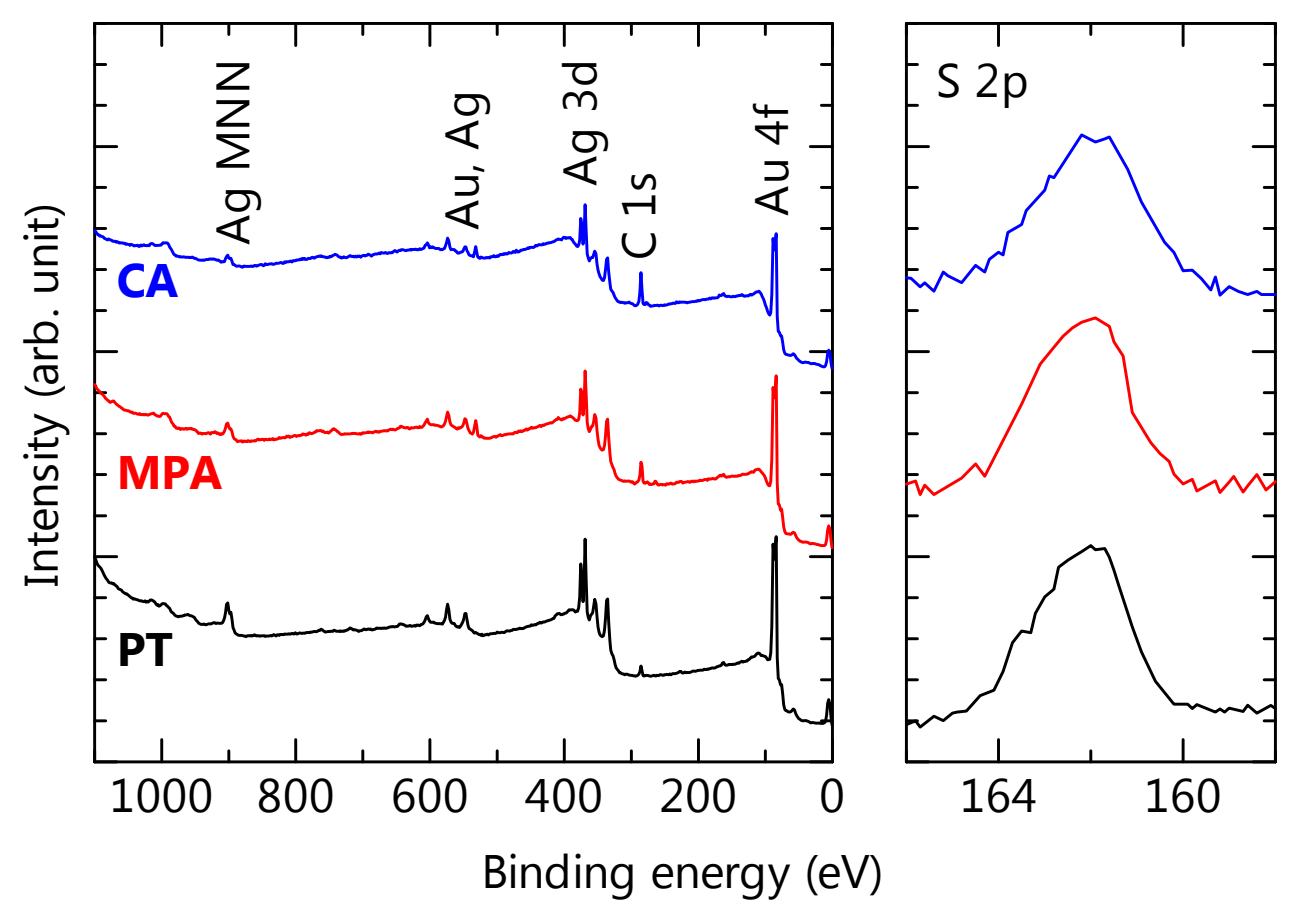

Fig. S1. XPS profiles of nanoporous gold modified by $10 \mathrm{mmol} / \mathrm{L}$ ethanolic solution of propanethiol (PT), mercaptopropionic acid (MPA) and cysteamine (CA). 